\title{
RÉPONSE AU STRESS INDUIT PAR LE TRANSPORT CHEZ LA TRUITE ARC-EN-CIEL (ONCORHYNCHUS MYKISS).
}

\author{
Y. ROUGER, J. AUBIN, B. BRETON, B. FAUCONNEAU, A. FOSTIER, \\ P.Y. LE BAIL, M. LOIR, P. PRUNET et G. MAISSE
}

INRA, Laboratoire de Physiologie des Poissons, IFR 43, Campus de Beaulieu, 35042 Rennes Cedex, France.

\section{RÉSUMÉ}

La réponse au stress induit par le transport a été étudiée chez des truites arc-en-ciel immatures d'un poids moyen de $200 \mathrm{~g}$. D'une manière globale, les valeurs de l'hématocrite, de l'osmolarité du plasma sanguin, du cortisol et de la testostérone sont significativement plus élevées chez les poissons transportés que chez les témoins non transportés, alors que les niveaux de gonadostimuline (GTH1) et d'hormone de croissance ne diffèrent pas suivant les lots. L'analyse du comportement en "open field " des individus de chaque lot montre que les poissons transportés s'immobilisent en moyenne après un temps significativement plus long que les témoins. L'étude des performances zootechniques de chaque lot pendant les deux mois suivant l'expérience indique que le lot transporté récupère rapidement.

\section{RESPONSE OF RAINBOW TROUT (ONCORHYNCHUS MYKISS) TO TRANSPORT STRESS.}

\section{ABSTRACT}

Response of rainbow trout to transport stress has been studied in immature fish weighing $200 \mathrm{~g}$. Hematocrite, blood plasma osmolarity, cortisol and testosterone levels are significantly higher in transported fishes than in control. However, there is no change of gonadotropin (GTH1) and growth hormone plasma levels during and after the transport. Behaviour analysis in "open field " shows that the transported fishes became immobile later than the control. Zootechnical performance analysis of each group after two months shows that transported fishes recuperate quickly.

\section{INTRODUCTION}

L'appréciation de l'importance du bien-être de l'animal de rente, à toutes les étapes de son élevage, constitue aujourd'hui un facteur important du jugement et de l'attitude des consommateurs. La notion de bien-être animal est généralement définie en tant que satisfaction des besoins physiologiques et comportementaux. De mauvaises conditions d'élevage pour l'animal entraînent des modifications physiologiques profondes, impliquant l'axe hypothalamo-hypophyso-surrénalien (DANTZER, 1981), dont les conséquences sur les performances zootechniques sont loin d'être négligeables. Par ailleurs, des altérations de la réactivité ou des modifications du répertoire comportemental peuvent apparaître dans un environnement inadapté (VEISSIER, 1996). La filière aquacole est, elle aussi, concernée par cette interrogation sur le bien-être animal. Parmi les facteurs potentiels de 
stress aigu en salmoniculture intensive, le transport des truites vivantes est probablement l'un des plus importants avec le tri. Le transport peut être effectué entre deux sites piscicoles à différents stades de l'élevage (alevin, truitelle, géniteur) et, pour les poissons de taille commerciale, vers la chaîne d'abattage. Cette pratique, courante en salmoniculture, a fait l'objet de peu d'études précises concernant ses effets sur la physiologie des poissons. D'après ce que l'on connaît chez les mammifères, la question de connaître l'impact du stress sur la survie, la croissance, la reproduction et la qualité de la chair se pose après un transport de poissons vivants. Il est donc primordial de connaître la nature et l'importance des perturbations engendrées par les manipulations et le transport, si l'effet produit est ou non réversible et enfin, le temps de récupération nécessaire.

Au cours du transport et des opérations qui lui sont liées, de nombreux facteurs de stress interviennent : la capture dans le milieu d'élevage (BARTON et PETER, 1982), le chargement dans la cuve de transport (MAULE et al., 1988 ; SPECKER et SCHRECK, 1980), un séjour plus ou moins long, à l'obscurité, dans des conditions surdensitaires (PICKERING et POTTINGER, 1987 ; PICKERING et al., 1991), la dégradation de la qualité de l'eau (Mc DONALD et al., 1993), les mouvements du véhicule, et enfin le déchargement dans un nouveau milieu d'élevage. Pour limiter les conséquences du stress, certains auteurs ont proposé de saler l'eau à raison de 5 à 6 grammes de $\mathrm{NaCl}$ par litre (HATTINGH et al., 1975 ; TAYLOR et SOLOMON, 1979a ; NIKINMAA et al., 1983) ou d'ajouter un tranquillisant (TAYLOR et SOLOMON, 1979b).

La plupart des études sur le transport des poissons, précédemment citées, se sont intéressées uniquement à des indicateurs physiologiques de stress, en particulier les niveaux de cortisol plasmatique, qui ne représentent que très partiellement les éventuels impacts sur les différentes fonctions (CARMICHAEL, 1984). Jusqu'à présent, le comportement de l'animal et sa réponse émotionnelle n'étaient pas pris en compte. Notre objectif a été de considérer dans leur globalité l'ensemble des facteurs de stress liés au transport, dans l'étude de différentes réponses physiologiques et comportementales, à court et moyen termes, chez la truite arc-en-ciel.

\section{MATÉRIEL ET MÉTHODES}

Les truites (Oncorhynchus mykiss) immatures (souche MRW, âge $0_{+}$, poids moyen $200 \mathrm{~g}$ ) utilisées dans cette étude ont été élevées dans des bassins circulaires de 6 mètres de diamètre $\left(10 \mathrm{~kg} / \mathrm{m}^{3}\right)$, à la Salmoniculture Expérimentale du Drennec, INRA, en Bretagne, dans les conditions naturelles de photopériode et de température. Les poissons n'étaient pas nourris depuis la veille de l'expérimentation.

L'expérimentation a été réalisée le 30 novembre 1995 (température de l'eau : $9^{\circ} \mathrm{C}$ ). Dès le lever du jour, les poissons du lot " transport " ont été chargés dans un camion où ils ont été répartis dans 2 cuves obscures de 450 litres $(0,80 \mathrm{~m} \times 0,74 \mathrm{~m} \times 0,74 \mathrm{~m})$ emplies d'eau de la pisciculture, à raison de 200 poissons par cuve, soit environ $90 \mathrm{~kg} / \mathrm{m}^{3}$. Avant le départ, un renouvellement de l'eau par surverse a été réalisé pendant 15 minutes. Au cours du transport, les poissons ont été maintenus dans une eau oxygénée à l'aide de bouteilles d'oxygène sous pression et de diffuseurs dont le débit était d'un litre par minute. Le $\mathrm{pH}$, la température, le niveau de gaz carbonique (dosage acide-base) et le niveau d'azote ammoniacal, $\mathrm{NH}_{4}{ }^{+}$, (méthode de Nessler, spectrophotomètre Hach de terrain) ont été mesurés dans le bassin d'élevage avant la pêche et dans les cuves avant le départ, puis à chaque retour du camion, immédiatement avant le prélèvement des poissons, 3, 6, 9 et 12 heures après le début du transport. 
Simultanément, un lot, transféré dans les nouvelles conditions d'élevage sans avoir été transporté, a été constitué : 80 poissons ont été disposés dans 4 bassins d'élevage de $1 \mathrm{~m}^{2}(300 \mathrm{I})$, à raison de 20 individus par bassin $\left(13 \mathrm{~kg} / \mathrm{m}^{3}\right)$. Enfin, le reste des poissons (lot " contrôle ") a été maintenu dans le bassin d'élevage d'origine, dont la superficie était réduite de moitié par une grille afin de conserver la charge initiale et limiter les manipulations lors des prélèvements ultérieurs. Par la suite, ce dernier groupe a subi les mêmes échantillonnages que le lot " transport " et aux mêmes heures.

Un échantillonnage a été réalisé sur 10 poissons prélevés dans le bassin d'élevage immédiatement avant le chargement des cuves (lot "pêche "). Quatre échantillonnages ont eu lieu dans le lot "transport " après 3,6, 9 et 12 heures à partir de l'heure du chargement. Les deux premiers échantillonnages ont été réalisés dans la cuve $n^{\circ} 1$ (déchargée à 6 heures) et les deux derniers dans la cuve $n^{\circ} 2$ (déchargée à 12 heures). Entre chaque échantillonnage le camion a effectué un trajet routier d'environ $2 \mathrm{H} 30$. A chaque échantillonnage, 10 poissons de chacun des lots " contrôle " et " transport " ont été pêchés et transférés dans un bac de 160 litres contenant une solution de phénoxy-2-éthanol (10 $\mathrm{ml}$ par 10 litres d'eau) permettant une anesthésie rapide avant les prélèvements sanguins. Simultanément, 10 autres poissons des mêmes lots ont été transférés dans un bac de 160 litres alimenté par l'eau de la pisciculture, dans l'attente des tests en "open field", décrits ci-dessous. Seuls 6 poissons sur 10 ont alors été testés afin de limiter l'effet de la diminution du nombre de poissons dans le bac d'attente.

Aux temps 6 heures et 12 heures, 80 poissons transportés ont été transférés dans 4 bassins d'élevage $\left(1 \mathrm{~m}^{2}\right)$ à raison de 20 individus par bassin. 10 poissons d'un bassin de chaque lot ont été anesthésiés, comme précédemment, le lendemain du transport pour les prélèvements sanguins et l'estimation du taux de lipides musculaires réalisée à l'aide d'un "Torry Fat Metter" (Distell Industry Ltd). Six des 10 poissons restant dans ces bassins ont subi le test en " open field " selon le même protocole que celui utilisé la veille. La croissance des poissons des 3 autres bassins de chaque lot a été suivie pendant les deux mois qui ont suivi le transport.

Le sang a été prélevé à l'aide d'une seringue héparinée dans un délai n'excédant pas 5 minutes après la capture. L'hématocrite a été mesuré, pour chaque poisson, après centrifugation à $12000 \mathrm{~g}$ d'une aliquote contenue dans un tube capillaire. Le reste de chaque prélèvement sanguin a été centrifugé pendant 10 minutes à $2500 \mathrm{~g}\left(4^{\circ} \mathrm{C}\right)$ et le plasma a été conservé congelé $\left(-20^{\circ} \mathrm{C}\right)$ jusqu'aux dosages.

Les paramètres plasmatiques suivants ont été mesurés :

- la pression osmotique du plasma a été mesurée à l'aide d'un osmomètre à pression de vapeur, Wescor 5500 ;

- les niveaux plasmatiques de la somatotropine $(\mathrm{GH})$, de la gonadostimuline (GTH1) et de la testostérone ont été mesurés à l'aide des dosages radioimmunologiques décrits respectivement par LE BAIL et al. (1991), GOVOROUN et al. (1997) et FOSTIER et JALABERT (1986). Le cortisol a été dosé par la méthode décrite par GOMEZ et al. (1996) en utilisant un anticorps commercial (Steranti, UK).

L'état émotionnel des poissons transportés et non transportés a été apprécié grâce au test d'« open field ". Mis au point par HALL (1938) pour comparer le comportement émotionnel de divers rongeurs et utilisé par DOTY et DOTY (1967) pour examiner l'effet de stress après manipulation, ce test a été adapté pour les poissons par STEELE (1983). Le comportement de l'animal, placé seul pendant deux minutes dans une enceinte éclairée artificiellement, dont le fond est quadrillé, est enregistré au moyen d'un vidéoscope permettant une analyse ultérieure précise et rigoureuse. Ce test permet d'apprécier l'état 
émotionnel des poissons à partir du nombre de passages dans la case centrale du bassin ou dans les cases périphériques. Les individus passant le plus souvent dans la case centrale du dispositif sont considérés comme étant les moins émotifs. L'état émotionnel des poissons s'apprécie également par des réactions comportementales comme le délai d'immobilisation.

La signification des différences observées entre les valeurs de chaque paramètre a été analysée à l'aide du test $T$ de Student (paramètres plasmatiques et délai d'immobilisation le jour du transport), du test $U$ de Mann et Whitney (paramètres plasmatiques et délai d'immobilisation à chaque temps) et $\mathrm{X}^{2}$ (proportion de poissons passant au centre de l'، open field" dans chaque lot).

\section{RÉSULTATS}

\section{Evolution des paramètres physico-chimiques de l'eau au cours du transport}

Les mesures effectuées simultanément dans les deux cuves jusqu'à 6 heures n'ont présenté aucune différence entre cuves, quel que soit le paramètre considéré. La température a peu varié au cours du transport, s'élevant progressivement de $8,7^{\circ} \mathrm{C}$, au moment du chargement, à $9,7^{\circ} \mathrm{C}$ à la fin de l'expérimentation. A la même heure, la température de l'eau de la pisciculture était de $8,9^{\circ} \mathrm{C}$. La concentration en oxygène dans l'eau du bassin d'origine et dans les cuves au début du transport était de $10 \mathrm{mg} / \mathrm{l}(90 \%$ de saturation). Rapidement, au cours des 3 premières heures du transport, la valeur de ce paramètre a augmenté pour se stabiliser jusqu'à la fin de l'expérimentation à des valeurs voisines de $30 \mathrm{mg} / /$ ( $270 \%$ de saturation) (Figure 1). La concentration en gaz carbonique, non détectable dans les bassins d'élevage, a augmenté rapidement dans les cuves, passant de $9 \mathrm{mg} / \mathrm{l}$, au départ, à $26 \mathrm{mg} / \mathrm{l}$, après 9 heures (Figure 1). De la même manière, le niveau d'azote ammoniacal ( $\mathrm{NH}^{+}$), très faible dans l'eau du bassin d'origine et, au départ, dans l'eau des cuves, a augmenté de manière très importante et régulière au cours des 12 heures de transport, passant de 0,4 à 6,8 mg/l (Figure 1). Le pH de l'eau du bassin était de 6,9. Dès le départ, le $\mathrm{pH}$ de l'eau des cuves s'est stabilisé à 6,0 pour toute la durée du transport.

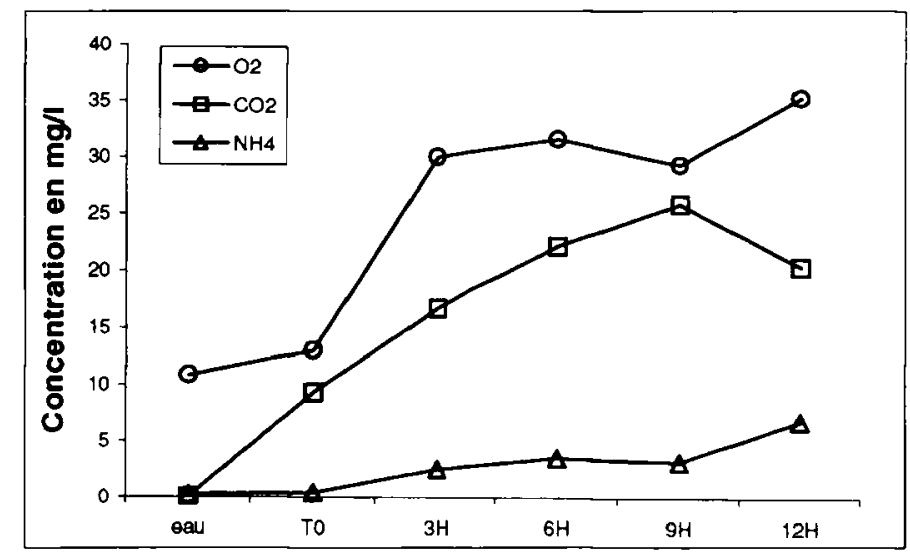

Figure 1

Concentrations en oxygène, gaz carbonique et azote ammoniacal dans l'eau de remplissage des cuves (" eau ") et évolution dans l'eau des cuves pendant le transport (T0, 3H, 6H, 9H, 12H).

\section{Figure 1}

Dissolved oxygen, carbon dioxide and ammonia levels in supply water (" eau ") and changes in tanks water during transport (T0, $3 \mathrm{H}, 6 \mathrm{H}, 9 \mathrm{H}, 12 \mathrm{H})$. 


\section{Analyse des effets du transport}

Tableau 1

Effet du transport sur la valeur de certains paramètres plasmatiques chez la truite arc-en-ciel immature.

Table I

Effect of transport on some plasma parameters levels of the immature rainbow trout.

\begin{tabular}{lccc}
\cline { 2 - 4 } & $\begin{array}{c}\text { Pêche } \\
(\mathbf{n}=10)\end{array}$ & $\begin{array}{c}\text { Contrôle } \\
(\mathbf{n = 4 0 )}\end{array}$ & $\begin{array}{c}\text { Transport } \\
(\mathbf{n}=40)\end{array}$ \\
\hline Hématocrite $(\%)$ & $41,3 \pm 1,14^{\mathrm{a}}$ & $40,4 \pm 0,51^{\mathrm{a}}$ & $45,5 \pm 0,64^{\mathrm{b}}$ \\
\hline Osmolarité $(\mathrm{mOsm} / \mathrm{kg})$ & $277 \pm 3,8^{\mathrm{a}}$ & $281 \pm 2,2^{\mathrm{a}}$ & $288 \pm 1,4^{\mathrm{b}}$ \\
\hline Cortisol $(\mathrm{ng} / \mathrm{ml})$ & $30,3 \pm 4,71^{\mathrm{a}}$ & $8,6 \pm 1,17^{\mathrm{b}}$ & $23,2 \pm 2,55^{\mathrm{a}}$ \\
\hline Testostérone $(\mathrm{ng} / \mathrm{ml})$ & $2,4 \pm 0,23^{\mathrm{a}}$ & $2,7 \pm 0,23^{\mathrm{a}}$ & $9,7 \pm 1,61^{\mathrm{b}}$ \\
\hline GTH1 $(\mathrm{ng} / \mathrm{ml})$ & $18,6 \pm 1,61^{\mathrm{a}}$ & $22,7 \pm 1,31^{\mathrm{a}}$ & $18,0 \pm 0,94^{\mathrm{a}}$ \\
\hline GH $(\mathrm{ng} / \mathrm{ml})$ & $0,44 \pm 0,09^{\mathrm{a}}$ & $0,68 \pm 0,10^{\mathrm{a}}$ & $0,47 \pm 0,07^{\mathrm{a}}$ \\
\hline
\end{tabular}

D'une manière globale (Tableau I), les valeurs de l'hématocrite, de l'osmolarité, du cortisol et de la testostérone sont significativement plus élevées pour les poissons transportés que pour les poissons du lot " contrôle " et aucune différence significative n'apparaît pour les valeurs de la GTH1 et de la GH. II est intéressant de noter la valeur élevée du cortisol plasmatique observée chez les individus pêchés le matin ; cette valeur est voisine de celle relevée chez les poissons transportés. La plupart de ces paramètres évolue peu après 3 heures de transport ; cependant, le niveau de testostérone plasmatique croît régulièrement au cours du transport pour atteindre un maximum après 9 heures, alors qu'il est stable chez les poissons du lot " contrôle » (Figure 2).

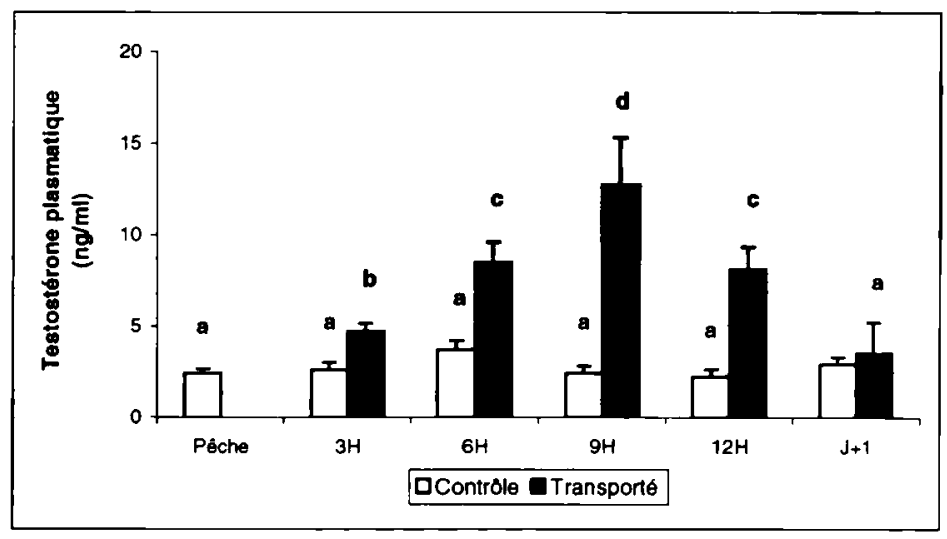

Figure 2

Evolution du niveau de testostérone plasmatique chez les truites arc-en-ciel immatures pendant le transport. Les moyennes marquées de la même lettre ne diffèrent pas significativement $(p=0,05)$.

Figure 2

Changes in the plasma testosterone level of immature rainbow trout during transport. Means marked by the same letter are not significantly different $(p=0.05)$. 
Les résultats de l'analyse comportementale en "open field " sont présentés dans le Tableau II. La proportion d'individus passant par le centre du dispositif ne varie pas significativement suivant les lots avec, cependant, une tendance à une fréquence plus élevée pour le lot " contrôle ". Le délai d'immobilisation est le paramètre le plus discriminant avec un temps de nage significativement supérieur pour les poissons transportés, quelle que soit la durée de transport.

\section{Tableau II}

Effet du transport sur le comportement de la truite arc-en-ciel immature en « open field".

\section{Table II}

Effect of transport on the behaviour in " open field" of the immature rainbow trout.

\begin{tabular}{lccc}
\cline { 2 - 4 } & $\begin{array}{c}\text { Pêche } \\
(\mathbf{n}=6)\end{array}$ & $\begin{array}{c}\text { Contrôle } \\
(\mathbf{n}=\mathbf{2 4})\end{array}$ & $\begin{array}{c}\text { Transport } \\
(\mathbf{n}=\mathbf{2 4})\end{array}$ \\
\hline Poissons passant au centre & $1 / 6^{\mathrm{a}}$ & $8 / 24^{\mathrm{a}}$ & $4 / 24^{\mathrm{a}}$ \\
\hline Délai d'immobilisation (s) & $33,8 \pm 9,5^{\mathrm{a}}$ & $38,9 \pm 8,0^{\mathrm{a}}$ & $64,4 \pm 8,8^{\mathrm{b}}$ \\
\hline
\end{tabular}

\section{Analyse de la récupération le lendemain du transport}

Le lendemain du transport, il n'existe plus de différence significative entre les valeurs des différents paramètres plasmatiques et comportementaux suivant les lots (Tableaux III et IV). II faut noter cependant les valeurs relativement élevées de l'hématocrite et de l'osmolarité du plasma sanguin par rapport à la veille et l'absence quasi totale d'individus passant au centre de l' " open field ". Par ailleurs, l'analyse de la teneur lipidique du muscle par "Torry Fat Metter " indique une différence significative en faveur des poissons transportés (contrôle : $5 \pm 0,3 \%$, transport $6 \mathrm{H}: 7 \pm 0,6 \%$, transport $12 \mathrm{H}: 6 \pm 0,3 \%$ ). Enfin, l'étude des performances zootechniques pendant les deux mois suivant l'expérimentation n'a mis en évidence aucune différence entre les lots, tant pour la croissance que pour la survie (données non présentées).

\section{Tableau III}

Effet du transport sur la valeur de certains paramètres plasmatiques, chez la truite arc-en-ciel immature, mesurés le lendemain du transport.

\section{Table III}

Effect of transport on plasma parameters levels of the immature rainbow trout, measured the next day.

\begin{tabular}{lccc}
\cline { 2 - 4 } & $\begin{array}{c}\text { Contrôle } \\
(\mathbf{n}=\mathbf{1 0})\end{array}$ & $\begin{array}{c}\text { Transport 6H } \\
(\mathbf{n = 1 0 )}\end{array}$ & $\begin{array}{c}\text { Transport 12H } \\
(\mathbf{n}=\mathbf{1 0})\end{array}$ \\
\hline Hématocrite $(\%)$ & $43,9 \pm 1,47^{\mathrm{a}}$ & $43,3 \pm 0,76^{\mathrm{a}}$ & $44,7 \pm 0,78^{\mathrm{a}}$ \\
\hline Osmolarité $(\mathrm{mOsm} / \mathrm{kg})$ & $304 \pm 2,5^{\mathrm{a}}$ & $286 \pm 3,0^{\mathrm{a}}$ & $299 \pm 2,6^{\mathrm{a}}$ \\
\hline Cortisol $(\mathrm{ng} / \mathrm{ml})$ & $15,2 \pm 3,82^{\mathrm{a}}$ & $14,1 \pm 2,89^{\mathrm{a}}$ & $28,1 \pm 6,51^{\mathrm{a}}$ \\
\hline Testostérone $(\mathrm{ng} / \mathrm{ml})$ & $2,9 \pm 0,37^{\mathrm{a}}$ & $3,7 \pm 0,81^{\mathrm{a}}$ & $3,5 \pm 1,70^{\mathrm{a}}$ \\
\hline GTH1 $(\mathrm{ng} / \mathrm{ml})$ & $16,4 \pm 2,44^{\mathrm{a}}$ & $15,6 \pm 2,40^{\mathrm{a}}$ & $16,2 \pm 1,93^{\mathrm{a}}$ \\
\hline GH $(\mathrm{ng} / \mathrm{ml})$ & $1,05 \pm 0,23^{\mathrm{a}}$ & $0,36 \pm 0,13^{\mathrm{a}}$ & $0,73 \pm 0,19^{\mathrm{a}}$ \\
\hline
\end{tabular}




\section{Tableau IV}

Effet du transport sur le comportement de la truite arc-en-ciel en " open field" observé le lendemain du transport.

\section{Table IV}

Effect of transport on the behaviour in " open field " of the immature rainbow trout, observed the next day.

\begin{tabular}{lccc}
\cline { 2 - 4 } & $\begin{array}{c}\text { Contrôle } \\
(\mathbf{n}=6)\end{array}$ & $\begin{array}{c}\text { Transport 6H } \\
(\mathbf{n}=6)\end{array}$ & $\begin{array}{c}\text { Transport 12H } \\
(\mathbf{n}=6)\end{array}$ \\
\hline Poissons passant au centre & $0 / 6^{\mathrm{a}}$ & $1 / 6^{\mathrm{a}}$ & $0 / 6^{\mathrm{a}}$ \\
\hline Délai d'immobilisation (s) & $34,8 \pm 18,4^{\mathrm{a}}$ & $34,2 \pm 17,5^{\mathrm{a}}$ & $23,0 \pm 9,2^{\mathrm{a}}$ \\
\hline
\end{tabular}

\section{DISCUSSION - CONCLUSION}

Les niveaux de cortisol élevés observés chez les poissons du lot pêché au petit jour, juste avant le transport, sont de l'ordre des valeurs maximales rapportées par GOMEZ et al. (1996), pendant la nuit, chez des truites arc-en-ciel équipées d'un cathéter pour la prise de sang. Ces niveaux pourraient donc refléter aussi bien des variations naturelles qu'un premier stress lié à la pêche. Ce stress, qui existe probablement, a un effet de courte durée puisque les poissons du lot "contrôle " présentent des faibles valeurs de cortisol plasmatique et il est sûrement très inférieur à celui provoqué par le chargement dans les cuves, que nous n'avons pas analysé. Pendant le transport, la densité élevée, le nonrenouvellement de l'eau et l'apport d'oxygène pur modifient considérablement la qualité de l'eau des cuves comme cela avait été montré par TAYLOR et SOLOMON (1979a) et BARTON et PETER (1982). Cependant, l'augmentation considérable de la teneur en $\mathrm{CO}_{2}$ semble ici compensée par le maintien d'une très forte concentration en oxygène et la concentration en ammoniac non jonisé toxique reste faible compte tenu de la valeur basse du $\mathrm{pH}(6)$ et de la température qui demeure inférieure à $10^{\circ} \mathrm{C}$ (EMERSON et al., 1975), malgré une constante élévation de l'azote ammoniacal total. Ces conditions, bien que compatibles avec la survie des poissons (aucune mortalité n'a été notée pendant l'expérience), apparaissent suffisamment différentes des conditions d'élevage pour induire un stress, révélé par des valeurs élevées de l'hématocrite et du niveau de cortisol plasmatique, comme cela avait déjà été observé par BARTON et PETER (1982) et MC DONALD et al. (1993). Les niveaux de cortisol observés pendant le transport restent inférieurs à $40 \mathrm{ng} / \mathrm{ml}$. Cette observation, comparée à celles de PICKERING et al. (1991) qui rapportent des niveaux de l'ordre de $80 \mathrm{ng} / \mathrm{ml}$ dans des expériences de confinement chez la même espèce, suggère que le stress subi dans nos conditions par les poissons transportés reste modéré. PICKERING et POTTINGER (1987) ont montré que la dégradation de la qualité de l'eau (faible concentration en oxygène, fortes teneurs en $\mathrm{CO}_{2}$ et $\mathrm{NH}^{-}$) pouvait diminuer l'amplitude de l'augmentation du cortisol chez la truite commune (Salmo trutta) en situation de confinement, mais cet effet disparaît lorsque le taux d'oxygène dissous est contrôlé et il est donc peu probable que nos observations correspondent à ce phénomène. L'analyse du niveau de testostérone plasmatique pendant un transport n'avait pas été réalisée avant notre étude. PICKERING et al. (1987) et PANKHURST et DEDUAL (1994) observent une baisse du niveau de testostérone chez des individus matures, respectivement de truite commune et de truite arc-en-ciel, soumis à des manipulations et au confinement. A l'inverse, avec des poissons immatures dans les conditions d'un transport, nous observons une augmentation régulière de la testostérone jusqu'à 9 heures. L'hypothèse de l'effet de l'augmentation de la densité, $\left(90 \mathrm{~kg} / \mathrm{m}^{3}\right.$ dans les 
cuves par rapport à $10 \mathrm{~kg} / \mathrm{m}^{3}$ dans le bassin d'élevage), et donc des interactions entre individus, sur le niveau de testostérone plasmatique est posée et devrait être testée en tenant compte de la durée, car dans nos conditions on observe une diminution de la testostérone après 12 heures de transport, ce qui laisse présager une adaptation à la nouvelle densité. L'analyse des paramètres comportementaux dans le test "open field " s'est avérée délicate en raison d'une très grande variabilité. Cependant, la proportion d'individus passant au centre du dispositif a été légèrement plus élevée dans le lot " contrôle " que dans les autres lots, ce qui est cohérent avec les hypothèses de départ. Le délai d'immobilisation est le paramètre le plus discriminant, les poissons transportés nageant plus longtemps que ceux du lot " contrôle ". Si l'on rapproche cette observation des résultats obtenus pour la testostérone plasmatique, l'hypothèse d'une plus grande agressivité des poissons transportés pourrait être envisagée.

L'état des poissons le lendemain du transport et le suivi de leurs performances zootechniques pendant les deux mois suivants montrent que les conditions du transport n'ont eu qu'un effet physiologique transitoire, sans perturbation à moyen terme, après retour dans la même eau d'élevage. En conclusion, il semble difficile de supprimer le stress induit par la pêche et le chargement des poissons qui est considéré comme le plus important par BARTON et PETER (1982). Par contre, il vaut mieux cerner les facteurs de perturbation liés au transport proprement dit. En particulier, il serait intéressant d'étudier les effets du facteur "augmentation de densité "sur le niveau de testostérone indépendamment du facteur "qualité d'eau ", puis de voir si l'utilisation d'un anesthésique à faible dose modifie la réponse, si celle-ci est confirmée.

\section{REMERCIEMENTS}

Cette étude a été réalisée avec la collaboration technique de $M$. Heydorff, A. Quemeneur, C. Gallais et L. Goardon et financée par I'INRA dans le cadre de l'A.I.P. "Bien-être et Sciences du Comportement".

\section{BIBLIOGRAPHIE}

BARTON B.A., PETER R.E., 1982. Plasma cortisol stress response in fingerling rainbow trout, Salmo gairdneri Richardson, to various transport conditions, anesthesia, and cold shock. J. Fish Biol., 20, 39-51.

CARMICHAEL G.J., 1984. Long distance truck transport of intensely reared large mouth bass. Progr. Fish-Cult., 46, 111-115.

DANTZER R., 1981. Physiologie du stress. La Recherche, 12 (120), p. 238.

DOTY L.A., DOTY B., 1967. Effects of handling at various ages on later open field behaviour. Can. J. Psychol., 21, 6 p.

EMERSON K., RUSSO R.C., LUND R.E., THURSTON R.V., 1975. Aqueous ammonia equilibrium calculations : effect of $\mathrm{pH}$ and temperature. J. Fish. Res. Board Can., 32, 2379-2383.

FOSTIER A., JALABERT B., 1986. Stereoidogenesis in rainbow trout (Salmo gairdnerl) at various preovulatory stages : changes in plasma hormone level and in vivo responses of the ovary to salmon gonadotropin. Fish Physiol. Biochem., 2, 87-99.

GOMEZ J.M., BOUJARD T., FOSTIER A., LE BAIL P.Y., 1996. Characterization of growth hormon nyctemeral plasma profiles in catheterized rainbow trout, Oncorhynchus mykiss. J. Exp. Zool., 274, 171-180.

GOVOROUN M.S., HUET J.C., PERNOLLET J.C., BRETON B., 1997. Use of immobilized metal ion affinity chromatography and dye-ligand chromatography for the separation 
and purification of rainbow trout pituitary gonadotropins, GTH-I and GTH-II. Chromatography $B$, (sous presse).

HALL C.S., 1938. The inheritance of emotionality. Sigma Xi Quart., 26, 17-27.

HATTINGH J., LE ROUX FOURIE F., VAN VUREN J.H.J., 1975. The transport of fresh water fish. J. Fish Biol., 7, 447-449.

LE BAIL P.Y., SUMPTER J.P., CARRAGHER J.F., MOUROT B., NIU P.D., WEIL C., 1991. Development and validation of a highly sensitive radioimmunoassay for chinook salmon (Oncorhynchus tshwytsha) growth hormone. Gen. Comp. Endocrinol., 83, 75-85.

Mc DONALD D.G., GOLDSTEIN M.D., MITTON C., 1993. Responses of hatchery-reared brook trout, lake trout, and splake to transport stress. Trans. Amer. Fish. Soc., 122, 1127-1138.

MAULE A.G., SCHRECK C.B., BRADFORD C.S., BARTON B.A., 1988. Physiological effects of collecting and transporting emigrating juvenile chinook salmon past dams on the Columbia river. Trans. Amer. Fish. Soc., 117, 245-261.

NIKINMAA M., SOIVIO A., NAKARI T., LINDGREN S., 1983. Hauling stress in brown trout (Salmo trutta) : physiological responses to transport in fresh water or salt water, and recovery in natural brackish water. Aquaculture, 34, 93-99.

PANKHURST N.W., DEDUAL M., 1994. Effects of capture and recovery on plasma levels of cortisol, lactate and gonadal steroids in natural population of rainbow trout. J. Fish Biol., 45, 1013-1025.

PICKERING A.D., POTTINGER T.G., 1987. Poor water quality suppresses the cortisol response of salmonid fish to handling and confinement. J. Fish Biol., 30, 363-374.

PICKERING A.D., POTTINGER T.G., CARRAGHER J., SUMPTER J.P., 1987. The effects of acute and chronic stress on the levels of reproductive hormones in the plasma of mature male brown trout, Salmo trutta L. Gen. Comp. Endocrinol., 68, 249-259.

PICKERING A.D., POTTINGER T.G., SUMPTER J.P., CARRAGHER J.F., LE BAIL P.Y., 1991. Effects of acute and chronic stress on the levels of circulating growth hormone in the rainbow trout, Oncorhynchus mykiss. Gen. Comp. Endocrinol., 83, 86-89.

SPECKER J.L., SCHRECK C.B., 1980. Stress responses to transportation and fitness for marine survival in coho salmon (Oncorhynchus kisutch) and steelhead trout (Salmo gairdneri). Can. J. Fish. Aquat. Sci., 37, 765-769.

STEELE C.W., 1983. Open field exploratory behaviour of fish ; An underutilized tool for behavioral toxicology. Marine Pollution Bulletin, 14, 124-125.

TAYLOR A.L., SOLOMON D.J., 1979a. Critical factors in the transport of living freshwater fish - I. General considerations and atmospheric gases. Fish. Mgmt., 10, 27-32.

TAYLOR A.L., SOLOMON D.J., 1979b. Critical factors in the transport of living freshwater fish - II. State feeding and ammonia excretion. Fish. Mgmt., 10, 81-85.

VEISSIER I., 1996. Utilisation du comportement pour évaluer les relations de l'animal avec son environnement. Colloque "Le bien-être des animaux d'élevage ; enjeux, approches, perspectives ", MORMEDE P., DANTZER R., LE NEINDRE P., (eds), 2-3 mai 1996, Université de Bordeaux 2. (Résumé). 